Research Paper

\title{
Outcome and Prospective Factor Analysis of High-dose Therapy Combined with Autologous Peripheral Blood Stem Cell Transplantation in Patients with Peripheral T-cell Lymphomas
}

Meng Wu, Xiaopei Wang, Yan Xie, Weiping Liu, Chen Zhang, Lingyan Ping, Zhitao Ying, Lijuan Deng, Wen Zheng, Ningjing Lin, Meifeng Tu, Yuqin Song, Jun Zhu凶

Key laboratory of Carcinogenesis and Translational Research (Ministry of Education), Department of Lymphoma, Peking University Cancer Hospital \& Institute

$\triangle$ Corresponding author: E-mail address: zhu-jun2017@outlook.com. Postal address: No 52, Fucheng Road, Haidian District, Beijing, China. 100142

(c) Ivyspring International Publisher. This is an open access article distributed under the terms of the Creative Commons Attribution (CC BY-NC) license (https://creativecommons.org/licenses/by-nc/4.0/). See http://ivyspring.com/terms for full terms and conditions.

Received: 2017.09.28; Accepted: 2018.04.12; Published: 2018.06.04

\begin{abstract}
Background: For peripheral T-cell lymphomas (PTCLs) patients, high-dose therapy combined with autologous peripheral blood stem cell transplantation (HDT/ASCT) has been an alternative treatment option, due to the lack of efficacy from conventional chemotherapy. While not all PTCLs could have benefit in survival from HDT/ASCT. The aim of this study was to evaluate the value of high-dose therapy combined with autologous peripheral blood stem cell transplantation (HDT/ASCT) in Chinese patients with Peripheral T-cell Lymphomas (PTCLs), in order to determine the cohort most suitable to receive HDT/ASCT.
\end{abstract}

Methods: A total of 79 patients with PTCLs who received HDT/ASCT in Peking University Cancer Hospital \& Institute from January 2001 to august 2016 were retrospectively analyzed.

Results: At a median follow-up time of 23.6 months, the 2-year progression-free survival (PFS) and 2-year overall survival (OS) of the entire cohort were $75.2 \%$ and $83.6 \%$ respectively. Patients with first complete remission (CRI) (2-year PFS 85.8\%, 2-year OS 94.2\%) were superior to others in survival. Patients with second complete remission (CR2) had no advantage in survival compared with those with first partial remission (PR1) (2-year PFS: $43.8 \%$ vs. $76.2 \%, p=0.128$; 2 -year OS: $72.9 \%$ vs. $77.1 \%, p=0.842$ ). In multivariate analysis, response before HDT/ASCT $(p=0.001)$ and LDH before HDT/ASCT $(p=0.047)$ were highly predictive for PFS, while no factors could independently predict OS. Subgroup analysis revealed that HDT/ASCT could improve the survival of patients with angioimmunoblastic T-cell lymphoma (AITL), especially in patients with chemosensitivity. Patients with natural killer / T-cell lymphoma (NKTCL) who received HDT/ASCT with CRI also had benefit in survival from HDT/ASCT, while nearly $90 \%$ of non-CRI patients appeared bone marrow involvement after HDT/ASCT.

Conclusion: Patients who achieved complete remission after first-line therapy, especially with AITL and NKTCL, should strongly be recommended to receive HDT/ASCT. The future prospective trial is warranted.

Key words: Peripheral T-cell lymphomas; High dose chemotherapy; Autologous peripheral blood stem cell transplantation; Retrospective study

\section{Introduction}

Peripheral T-cell lymphomas (PTCLs) are a heterogeneous group of lymphomas. It accounts for approximately $10 \%$ of non-Hodgkin lymphomas cases.[1] The proportion is higher in Asian than in western countries, about $20-25 \%$, mainly due to a higher incidence rate of natural killer/T-cell lymphoma (NKTCL) and adult T-cell lymphoma/ leukemia (ATLL).[2-4] Anthracycline-based regimens, such as CHOP (cyclophosphamide, doxorubicin, vincristine, prednisone) or CHOPE (CHOP + etoposide), are the preferred choice of frontline treatment for most subtypes of PTCLs,[5] albeit treatment outcomes from PTCLs are much less satisfactory than outcomes from aggressive B cell 
lymphomas.[6] The 5-year overall survival (OS) rates vary among different pathological subtypes, which in general are below 40\%.[2] The activity of L-asparaginase-containing regimens is well established for NKTCL.[7] However, the 5-year OS rates of nasal NKTCL and extranasal NKTCL are only $42 \%$ and $9 \%$ respectively.[2]

The lack of efficacy from conventional therapy prompted many to attempt high-dose therapy combined with autologous peripheral blood stem cell transplantation (HDT/ASCT). HDT/ASCT had been a reasonable treatment option for PTCLs patients in both consolidation and salvage therapy. Hitherto, there has been no randomized controlled trial assessing the clinical value of HDT/ASCT in combination with conventional therapy, due to the low incidence rate and diverse pathological subtypes of PTCLs. The latest meta-analysis showed that the efficacy of HDT/ ASCT in PTCLs varied greatly from study to study. [8] The high heterogeneity was mainly due to the different design of clinical trials (prospective or retrospective trials), pathological subtypes proportions, and disease status before HDT/ASCT. It indicated that not all PTCLs could have benefit in survival from HDT/ASCT. We therefore carried out a retrospective analysis of 79 cases to evaluate the value of HDT/ASCT in Chinese patients with PTCLs, in order to determine the cohort most suitable to receive HDT/ASCT.

\section{Patients and methods}

\section{Patients}

A total of 79 patients with PTCLs were retrospectively analyzed. These patients received HDT/ASCT at Peking University Cancer Hospital \& Institute from January 2001 to August 2016. Their histopathologic data were identified by the pathology department according to "the 2008 WHO classification of tumors of hematopoietic and lymphoid tissues".[9] Patients with mature T-cell and natural killer-cell neoplasms, except those with primary cutaneous lymphoma and leukemia, were included in the study. The analysis was approved by the Medical Ethics Committee of Peking University Cancer Hospital \& Institute.

\section{HDTIASCT protocol}

According to intention-to-treat, patients who qualifed HDT/ASCT could receive mobilization regimen after 3-4 cycles of first-line treatment or 1-2 cycles of salvage treatment. Prior to mobilization, we verified the absence of lymphoma involvement by bone marrow biopsy. In addition to granulocyte colony stimulating factor (G-CSF), mobilization was achieved with cyclophosphamide-based regimen (57 cases), with high-dose methotrexate regimen (4 cases), with high-dose cytosine arabinoside (7 cases), with gemcitabine-based regimen ( 3 cases), and (4 cases) another 2 cases with drug in clinical trial (Mozobil/placebo).

After the whole first-line or salvage treatment, patients who were ready for HDT/ASCT received conditioning regimen followed by peripheral blood stem cell infusion. The conditioning regimen included 47 cases with CBV (cyclophosphamide, carmustine, etoposide), 10 cases with BEAC (carmustine, etoposide, cytosine arabinoside, cyclophosphamide), 20 cases with BEAM (carmustine, etoposide, cytosine arabinoside, melphalan), and 2 cases with total-body irradiation (TBI) and high-dose cyclophosphamide (HD-CTX), followed by peripheral blood stem cell infusion.

\section{Efficacy assessment and follow-up criteria}

Tumor responses were assessed every other cycle of chemotherapy during first-line or salvage therapy, before HDT/ASCT, 6-8 weeks after HDT/ASCT, and every 6 to 12 months until disease progression. We adopted efficacy criteria reported by Cheson et al. [10] to assess responses to treatment for cases after 2007, and retrospectively for cases before 2007. All the patients were followed up from inpatients, outpatients or telephone. Follow up time was defined from the first day of high-dose therapy until death or the last follow-up.

\section{Statistic methods}

All data analyzed with IBM SPSS Statistics, version 20.0. Progression-free survival (PFS) was measured from the first day of high-dose therapy to the first relapse, progressive disease, or last follow-up. OS was calculated from the first day of high-dose therapy until death as a result of any cause. PFS and OS rates were estimated using the Kaplan-Meier method. Log-rank tests and Cox regression models were used to analyze the univariate and multivariate impacts of various prognostic factors. To identify prognostic variables for PFS and OS, univariate analysis was performed for the following clinical parameters: histologic subtypes, Eastern Cooperative Oncology Group performance status (ECOG PS), stage, B symptoms, age adjusted International Prognostic Index (aaIPI), Prognostic Index for T-cell lymphoma (PIT), disease status before transplantation, the level of Lactate dehydrogenase (LDH), Erythrocyte Sedimentation Rate (ESR), and Beta 2 microspheres $(\beta 2-\mathrm{MG})$ pretreatment, and the level of LDH, ESR, and $\beta 2-\mathrm{MG}$ before HDT/ASCT. Furthermore, event-free survival (EFS) at 24 months (EFS24) and subsequent overall survival (OSsub) were 
evaluated. EFS was defined as time between the date of diagnosis and progression after primary treatment, retreatment, or death. OSsub was measured after achieving EFS24 or from the time of progression if it occurred within 24 months.[11]

\section{Results}

\section{Clinicalpathologic features}

Pathological subtypes of the 79 patients included: ALCL $(\mathrm{n}=32,40.5 \%)$, PTCL not otherwise specified (PTCL-NOS, $\mathrm{n}=10,12.7 \%$ ), angioimmunoblastic T-cell lymphoma (AITL, n=14, 17.7\%), NKTCL $(\mathrm{n}=21,26.6 \%)$, subcutaneous panniculitis-like T-cell lymphoma- $\alpha \beta$ (SPTCL- $\alpha \beta, n=1,1.3 \%$ ), subcutaneous panniculitis-like T-cell lymphoma- $\gamma \delta$ (SPTCL- $\gamma \delta, n=1$, $1.3 \%)$. In the ALCL cohort, about one half $(18 / 32)$ were diagnosed as ALK-positive ALCL. More details of the clinicalpathologic features were listed in Table 1.

\section{Treatment prior to HDT/ASCT}

Patients received on average 8.3 cycles (4-30 cycles) of chemotherapy prior to HDT/ASCT. For first-line therapy, most non-NKTCL patients $(56 / 58)$ received $\mathrm{CHOP}$-like (the main drugs in CHOP-like regimen was cyclophosphamide, doxorubicin, vincristine, prednisone) regimens. L-asparaginase was administered in 18 out of $21(85.7 \%)$ NKTCL patients with NKTCL. The other 3 NKTCL patients who underwent treatment before 2009, did not receive L-asparaginase due to a lack of consensus about efficacy then. Local radiotherapy was used in 9/13 NKTCL patients who had nasal cavity involvement. A total of 47 patients were sensitive to first-line treatment and received HDT/ASCT as consolidation treatment. While the other 32 cases, who were refractory to first-line therapy or with relapse disease, received HDT/ ASCT after salvage chemotherapy.

Table 1. Pretherapeutic Clinicopathologic Patient Characteristics

\begin{tabular}{|c|c|c|c|c|c|c|}
\hline & \multicolumn{2}{|c|}{ Sensitivity to first-line treatment $(\mathrm{N}=47)$} & \multicolumn{2}{|c|}{ Sensitivity to salvage treatment $(\mathrm{N}=22)$} & \multicolumn{2}{|c|}{ Refractory disease $(\mathrm{N}=10)$} \\
\hline & $\mathrm{N}$ & $\%$ & $\mathrm{~N}$ & $\%$ & $\mathrm{~N}$ & $\%$ \\
\hline \multicolumn{7}{|l|}{ Sex } \\
\hline Male & 32 & $68.1 \%$ & 18 & $81.8 \%$ & 8 & $80.0 \%$ \\
\hline Female & 15 & $31.9 \%$ & 4 & $18.2 \%$ & 2 & $20.0 \%$ \\
\hline Median age of on set (range)/year & \multicolumn{2}{|l|}{$35(9-60)$} & \multicolumn{2}{|l|}{$29(14-61)$} & \multicolumn{2}{|c|}{$35(14-53)$} \\
\hline \multicolumn{7}{|l|}{ Subtype of pathology } \\
\hline ALCL ALK+ & 12 & $25.5 \%$ & 5 & $22.7 \%$ & 1 & $10.0 \%$ \\
\hline ALCL ALK- & 6 & $12.8 \%$ & 2 & $9.1 \%$ & 1 & $10.0 \%$ \\
\hline ALCL ALK unknown & 3 & $6.4 \%$ & 2 & $9.1 \%$ & 0 & $0.0 \%$ \\
\hline PTCL-NOS & 4 & $8.5 \%$ & 3 & $13.6 \%$ & 3 & $30.0 \%$ \\
\hline AITL & 10 & $21.3 \%$ & 3 & $13.6 \%$ & 1 & $10.0 \%$ \\
\hline NKTCL & 12 & $25.5 \%$ & 5 & $22.7 \%$ & 4 & $40.0 \%$ \\
\hline SPTCL- $\alpha \beta$ & 0 & $0.0 \%$ & 1 & $4.5 \%$ & 0 & $0.0 \%$ \\
\hline SPTCL- $\gamma \delta$ & 0 & $0.0 \%$ & 1 & $4.5 \%$ & 0 & $0.0 \%$ \\
\hline \multicolumn{7}{|l|}{ Stage } \\
\hline Stage I & 1 & $2.1 \%$ & 3 & $13.6 \%$ & 0 & $0.0 \%$ \\
\hline Stage II & 10 & $21.3 \%$ & 5 & $22.7 \%$ & 1 & $10.0 \%$ \\
\hline Stage III & 8 & $17.0 \%$ & 3 & $13.6 \%$ & 3 & $30.0 \%$ \\
\hline Stage IV & 28 & $59.6 \%$ & 11 & $50.0 \%$ & 6 & $60.0 \%$ \\
\hline B Symptom & 29 & $61.7 \%$ & 7 & $31.8 \%$ & 5 & $50.0 \%$ \\
\hline Extranodal sites $>1$ & 21 & $44.7 \%$ & 8 & $36.4 \%$ & 5 & $50.0 \%$ \\
\hline \multicolumn{7}{|l|}{ ECOG PS } \\
\hline 0 & 29 & $61.7 \%$ & 13 & $59.1 \%$ & 6 & $60.0 \%$ \\
\hline 1 & 14 & $29.8 \%$ & 9 & $40.9 \%$ & 4 & $40.0 \%$ \\
\hline 2 & 4 & $8.5 \%$ & 0 & $0.0 \%$ & 0 & $0.0 \%$ \\
\hline \multicolumn{7}{|c|}{$\begin{array}{l}\text { The data below excluded } 22 \text { cases whose LDH data before treatment could not be collected. } \\
\qquad(\mathrm{N}=34)\end{array}$} \\
\hline LDH elevated before treatment $(\mathrm{LDH}>240 \mathrm{U} / \mathrm{L})$ & 18 & $52.9 \%$ & 3 & $20.0 \%$ & 5 & $62.5 \%$ \\
\hline \multicolumn{7}{|l|}{ aaIPI } \\
\hline 0 & 3 & $8.8 \%$ & 4 & $46.7 \%$ & 0 & $0.0 \%$ \\
\hline 1 & 13 & $38.2 \%$ & 9 & $60.0 \%$ & 4 & $50.0 \%$ \\
\hline 2 & 17 & $50.0 \%$ & 2 & $13.3 \%$ & 4 & $50.0 \%$ \\
\hline Age $>60$ & 1 & $2.9 \%$ & - & - & - & - \\
\hline \multicolumn{7}{|l|}{ PIT } \\
\hline 0 & 14 & $41.2 \%$ & 15 & $71.4 \%$ & 2 & $25.0 \%$ \\
\hline 1 & 17 & $50.0 \%$ & 6 & $28.6 \%$ & 5 & $62.5 \%$ \\
\hline 2 & 3 & $8.8 \%$ & 0 & $0.0 \%$ & 1 & $12.5 \%$ \\
\hline
\end{tabular}

* There were 22 patients whose LDH data before treatment were unavailable, such that aaIPI and PIT could only be calculated in the remaining 57 patients. Among the 57 patients, a total of 34 patients were sensitive to first line treatment, 15 patients were sensitive to salvage treatment, and the rest showed no response to chemotherapy before HDT/ASCT.

ALCL: Anaplastic large-cell lymphoma; ALK+: Anaplastic lymphoma kinase expressing; ALK-: without anaplastic lymphoma kinase expressing; PTCL-NOS: Peripheral T-cell lymphoma, not otherwise specified; AITL: Angioimmunoblastic T-cell lymphoma; NKTCL: extranodal natural killer/T-cell lymphoma, nasal type; SPTCL: Subcutaneous panniculitis-like T-cell lymphoma; ECOG PS: Eastern Cooperative Oncology Group performance status; LDH: Lactate dehydrogenase; $\beta 2-$ MG: $\beta 2$-Microglobulin; ESR: Erythrocyte sedimentation rate; aaIPI: age adjusted International Prognostic Index; PIT: Prognostic Index for T-cell lymphoma. 
Their second-line therapy regimen was selected from one of the followings: CHOPE-like, DICE (dexamethasone, ifosfamide, cisplatin, etoposide), ICE (ifosfamide, carboplatin, etoposide), GDP (gemcitabine, cisplatin, prednison), Gemox (gemcitabine, oxaliplatin), and HD-MTX (high-dose methotrexate), depending on the effect of first-line therapy, interval between the end of first-line therapy and relapse, involved field, and the status of patients.

\section{Response to treatment before HDT/ASCT}

According to the response before HDT/ASCT, patients were categorized into five groups: first complete remission (CR1) (38 cases), first partial remission (PR1) (9 cases), complete remission after salvage therapy (CR2) (10 cases), partial remission after salvage therapy (PR2) (12 cases), and stable disease or progressive disease (SD/PD) (10 cases). HDT/ASCT served as consolidation treatment for patients sensitive to first-line treatment (group CR1+PR1, 47 cases), or as component of salvage treatment for relapse/refractory patients (group $\mathrm{CR} 2+\mathrm{PR} 2+\mathrm{SD} / \mathrm{PD}, 32$ cases).

\section{Response to HDT/ASCT}

Within 8 weeks after HDT/ASCT, seven patients showed rapid decrease of tumor load; eight patients had disease progression ( 5 cases with PR2 and 3 cases with SD/PD). The disease condition for the rest of the patients remained unchanged from their pre-HDT/ ASCT status.

Until the last follow-up, 59 patients survived, including 48 patients with CR. A total of 29 patients had disease progression after HDT/ASCT, 20 of which died as a result of progressive disease. Among the rest of the PD patients, three patients later achieved CR through chemotherapy or tandem HDT/ASCT, while six patients were still under therapy as of the writing of this report.

\section{Survival of the entire cohort after HDTIASCT}

With a median follow-up time of 23.6 months (range: 1.5-185.8), the 2-year PFS and 2-year OS of the entire cohort were $75.2 \%$ and $83.6 \%$ respectively (Figure 1). For patients who received HDT/ASCT as consolidation treatment, their 2-year PFS and OS were $83.8 \%$ and $89.8 \%$ respectively. When HDT/ASCT were used as salvage treatment, the resulting 2-year PFS and OS were $32.9 \%$ and $50.5 \%$ respectively. It is thus unsurprising that the response before HDT/ASCT strongly correlate with PFS and OS $(p<0.001)$ (Figure 2). Group CR1 (2-year PFS 85.8\%, 2-year OS $94.2 \%$ ) were superior to other groups in survival. Group PR1 showed advantage in PFS compared with group CR2 (2-year PFS: $76.2 \%$ vs. $43.8 \%, p=0.128)$ in spite of a lack of statistical significance. OS for group PR1 and group CR2 were similar (2-year OS: $77.1 \%$ vs. $72.9 \%, p=0.842$ ).

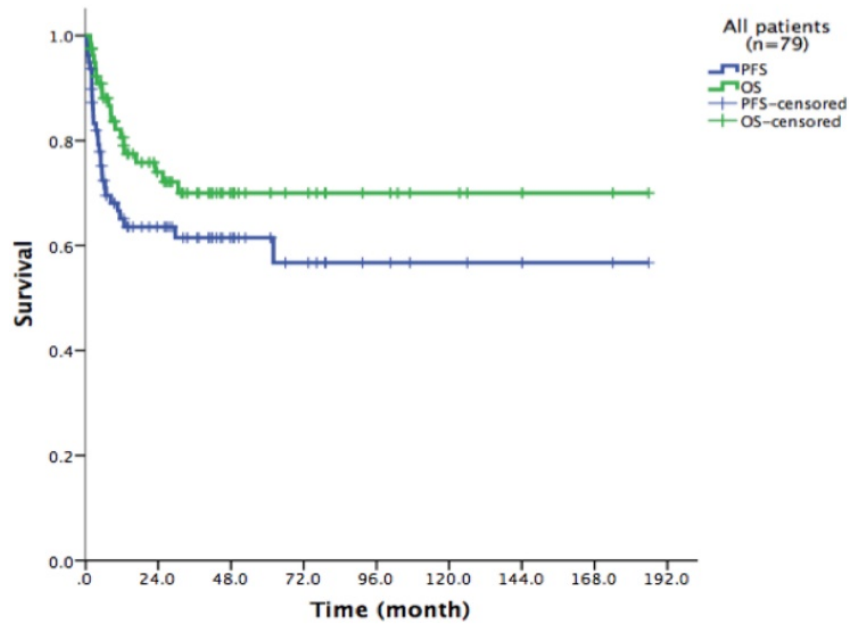

Fig 1. 79 patients Kaplan-Meier curve of overall survival and progression free survival
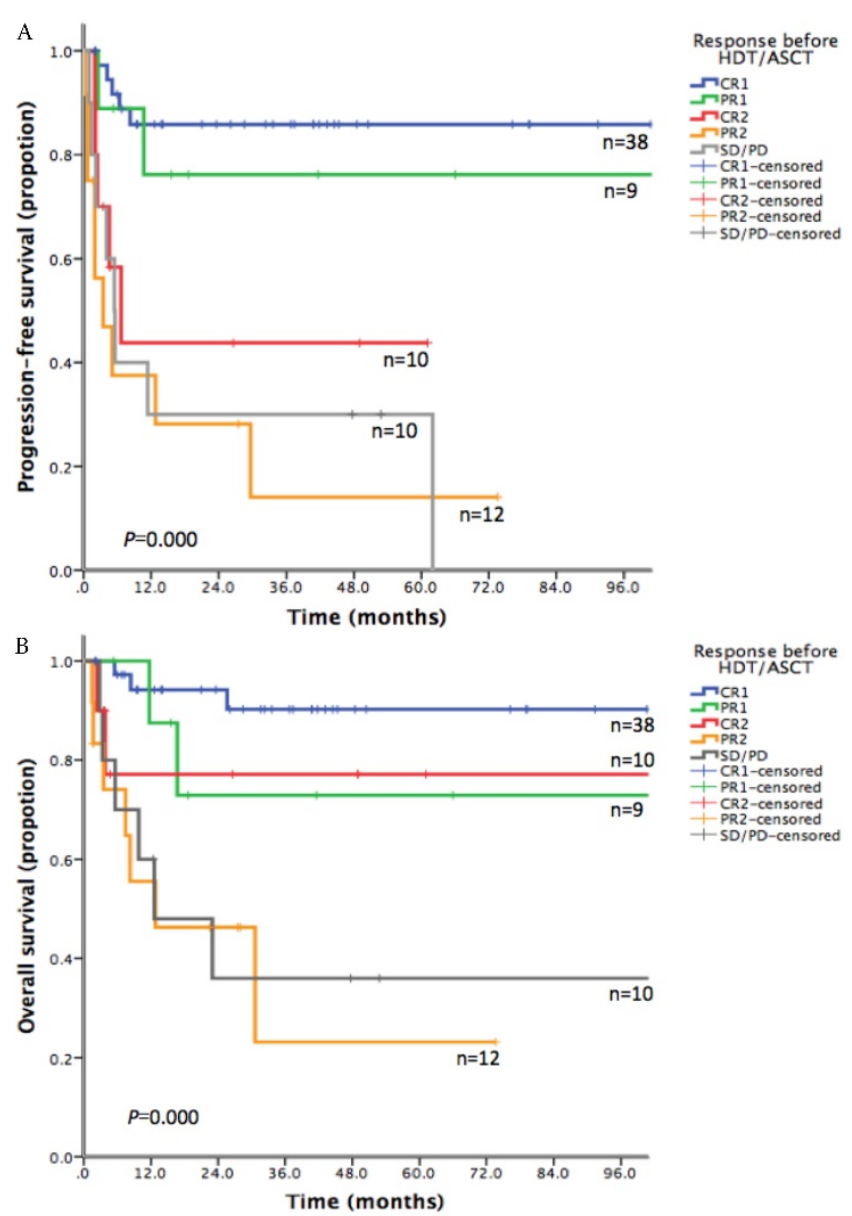

Fig 2. A. Progression free survival depending on response before HDT/ASCT in 79 PTCLs patients. B. Overall survival depending on response before HDT/ASCT in 79 PTCLs patients

\section{Survival of AITL and NKTCL subgroups after HDTIASCT}

In the subgroup analysis, all 14 patients with AITL were under 60 -year-old with ECOG $\leq 1$. Majority 
of patients $(13 / 14)$ presented with advanced stage. B symptom was noted in 5 cases. Two patients had more than one extranodal site. We were able to calculate PIT and aaIPI for 11 out of 14 cases (Table 2). The 2-year PFS and OS were $74.3 \%$ and $94.9 \%$ respectively in these patients.

Table 2. PIT, aalPI, and response before HDT/ASCT in AITL Patients

\begin{tabular}{lllcll}
\hline & $\mathrm{N}$ & $\%$ & $\mathrm{~N}$ & $\%$ \\
\hline PIT & & & Response before HDT/ASCT & & \\
\hline 0 & 4 & $28.6 \%$ & CR1 & 8 & $57.1 \%$ \\
1 & 6 & $42.9 \%$ & PR1 & 2 & $14.3 \%$ \\
2 & 1 & $7.1 \%$ & CR2 & 2 & $14.3 \%$ \\
Unknown & 3 & $21.4 \%$ & PR2 & 1 & $7.1 \%$ \\
aaIPI & & & SD/PD & 1 & $7.1 \%$ \\
0 & 0 & $0.0 \%$ & & & \\
1 & 4 & $28.6 \%$ & & & \\
2 & 7 & $50.0 \%$ & & & \\
Unknown & 3 & $21.4 \%$ & & &
\end{tabular}

PIT: Prognostic Index for T-cell lymphoma; aaIPI: age adjusted International Prognostic Index; CR1: complete remission after first-line treatment; PR1: partial remission after first-line treatment; CR2: complete remission after second-line treatment; PR2: partial remission after second-line treatment; SD: Stable disease PD: Progressive disease.

In NKTCL cohort, 9 patients received HDT/ASCT as salvage treatment, while the other 12 patients were sensitive to first-line treatment and underwent HDT/ASCT as consolidation treatment. More than half of patients $(13 / 21)$ presented with advanced stage. Extranasal NKTCL was accounted for $23.8 \%$ of this cohort $(5 / 21)$, which was similar to the number previously reported by the International Peripheral T-Cell Lymphoma Project.[12] The 2-year PFS and 2-year OS of the entire cohort were $54.5 \%$ and $60.0 \%$ respectively. Most patients with CR1 (9/11) kept long time survival until the last follow-up. In the rest ten patients with non-CR1, only three patients were still alive at the last follow-up. Disease progression in NKTCL patients usually occurred within 6 months (1.0-5.6 months) after HDT/ASCT, which lead to death within a year (2.3-12.6 months). Among the nine patients with $\mathrm{PD}$, the initial symptoms accompanied with disease progression include nasal obstruction (one), and fever combined with pancytopenia (eight). The latter eight patients were suspected of bone marrow involvement, two of which were confirmed by bone marrow biopsy.

\section{Prognostic parameters}

Based upon univariate analysis (Table 3), factors that strongly correlated with PFS included: LDH before HDTA/ASCT (2-year PFS 67.9\% vs. 34.1\%, $p=0.019$ ), ESR before HDT/ASCT (2-year PFS 79.8\% vs. $47.9 \%, p=0.003)$, and response before HDT/ASCT $(p<0.001)$. However, LDH and ESR before initial treatment did not show any correlation with PFS. Predictors of OS included response before

HDT/ASCT $(p<0.001), \quad \beta 2-\mathrm{MG}$ before HDT/ASCT (2-year OS: $80.7 \%$ vs. $57.1 \%, p=0.028$ ), ESR before initial treatment (2-year OS: $80.8 \%$ vs. $56.2 \%, p=0.035$ ), and ESR before HDT/ASCT (2-year OS: 91.3\% vs. $52.3 \%, p=0.001$ ). In multivariate analysis (Table 4), achieving CR1 before HDT/ASCT (Hazard rate (HR) $=0.230, p=0.001)$ and LDH before HDT/ASCT $(\mathrm{HR}=3.64, p=0.047)$ were highly predictive of PFS, while no factors could independently predict OS.

Table 3. Prognostic factors in univariate analysis

\begin{tabular}{|c|c|c|c|c|c|}
\hline & \multirow[t]{2}{*}{$\mathrm{N}$} & \multicolumn{2}{|c|}{ 2-year PFS } & \multicolumn{2}{|c|}{ 2-year OS } \\
\hline & & $\%$ & $P$ & $\%$ & $P$ \\
\hline Sex & & & 0.135 & & 0.062 \\
\hline Male & 58 & 57.9 & & 73.5 & \\
\hline Female & 21 & 78.5 & & 88.4 & \\
\hline Subtype of pathology & & & 0.410 & & 0.538 \\
\hline ALCL ALK+ & 18 & 77.8 & & 83.0 & \\
\hline ALCL ALK- & 9 & 55.6 & & 64.8 & \\
\hline ALCL ALK unknown & 5 & 80.0 & & 100.0 & \\
\hline PTCL & 10 & 57.1 & & 64.8 & \\
\hline AITL & 14 & 74.3 & & 92.9 & \\
\hline NKTCL & 21 & 54.5 & & 60.1 & \\
\hline SPTCL- $\alpha \beta$ & 1 & 0.0 & & 0.0 & \\
\hline SPTCL- $\gamma \delta$ & 1 & 0.0 & & 100.0 & \\
\hline ECOG & & & 0.268 & & 0.269 \\
\hline 0 & 48 & 65.3 & & 76.8 & \\
\hline 1 & 27 & 55.5 & & 65.0 & \\
\hline 2 & 4 & 100.0 & & 100.0 & \\
\hline \multicolumn{6}{|l|}{ Stage } \\
\hline Stage I & 4 & 50.0 & 0.825 & 50.0 & 0.536 \\
\hline Stage II & 15 & 57.0 & & 67.9 & \\
\hline Stage III & 15 & 71.8 & & 86.2 & \\
\hline Stage IV & 45 & 64.0 & & 73.8 & \\
\hline B Symptom & & & 0.738 & & 0.743 \\
\hline No & 38 & 65.6 & & 76.6 & \\
\hline Yes & 41 & 61.8 & & 71.6 & \\
\hline Extranodal sites & & & 0.691 & & 0.862 \\
\hline$\leq 1$ & 45 & 67.4 & & 74.7 & \\
\hline$>1$ & 34 & 58.5 & & 72.9 & \\
\hline LDH before treatment & & & 0.393 & & 0.327 \\
\hline Normal ( $\leq 240 \mathrm{U} / \mathrm{L})$ & 31 & 58.4 & & 67.4 & \\
\hline Elevated $(>240 \mathrm{U} / \mathrm{L})$ & 26 & 63.8 & & 75.8 & \\
\hline Unknown & 22 & - & & - & \\
\hline$\beta 2-\mathrm{MG}$ before treatment & & & 0.420 & & 0.238 \\
\hline Normal $(\leq 3.0 \mathrm{mg} / \mathrm{L})$ & 35 & 63.1 & & 73.5 & \\
\hline Elevated $(>3.0 \mathrm{mg} / \mathrm{L})$ & 16 & 53.5 & & 62.7 & \\
\hline Unknown & 28 & - & & - & \\
\hline ESR before treatment & & & 0.140 & & 0.035 \\
\hline Normal $(\leq 15 \mathrm{~mm} / \mathrm{h})$ & 28 & 66.7 & & 80.8 & \\
\hline Elevated $(>15 \mathrm{~mm} / \mathrm{h})$ & 24 & 46.3 & & 56.2 & \\
\hline Unknown & 27 & - & & - & \\
\hline aaIPI & & & 0.537 & & 0.215 \\
\hline 0 & 7 & 57.1 & & 71.4 & \\
\hline 1 & 26 & 58.2 & & 61.5 & \\
\hline 2 & 23 & 67.2 & & 81.4 & \\
\hline LDH unknown or age $>60$ & 23 & - & & - & \\
\hline PIT & & & 0.770 & & 0.691 \\
\hline 0 & 27 & 59.5 & & 67.3 & \\
\hline 1 & 26 & 59.3 & & 75.4 & \\
\hline 2 & 4 & 75.0 & & 66.7 & \\
\hline Unknown & 22 & - & & - & \\
\hline Response before HDT/ASCT & & & $<0.001$ & & $<0.001$ \\
\hline CR1 & 38 & 85.8 & & 94.2 & \\
\hline PR1 & 9 & 76.2 & & 72.9 & \\
\hline CR2 & 10 & 43.8 & & 77.1 & \\
\hline PR2 & 12 & 28.1 & & 46.3 & \\
\hline $\mathrm{SD} / \mathrm{PD}$ & 10 & 30.0 & & 36.0 & \\
\hline LDH before HDT/ASCT & & & 0.019 & & 0.728 \\
\hline Normal $(\leq 240 \mathrm{U} / \mathrm{L})$ & 67 & 67.9 & & 74.6 & \\
\hline
\end{tabular}




\begin{tabular}{|c|c|c|c|c|c|}
\hline & \multirow{2}{*}{$\mathrm{N}$} & \multicolumn{2}{|c|}{ 2-year PFS } & \multicolumn{2}{|c|}{ 2-year OS } \\
\hline & & $\%$ & $P$ & $\%$ & $P$ \\
\hline Elevated (>240U/L) & 11 & 34.1 & & 65.5 & \\
\hline Unknown & 1 & - & & - & \\
\hline$\beta 2-\mathrm{MG}$ before HDT/ASCT & & & 0.146 & & 0.028 \\
\hline Normal $(\leq 3.0 \mathrm{mg} / \mathrm{L})$ & 55 & 68.8 & & 80.7 & \\
\hline Elevated $(>3.0 \mathrm{mg} / \mathrm{L})$ & 10 & 60.0 & & 57.1 & \\
\hline Unknown & 14 & - & & - & \\
\hline ESR before HDT/ASCT & & & 0.003 & & 0.001 \\
\hline Normal $(\leq 15 \mathrm{~mm} / \mathrm{h})$ & 36 & 79.8 & & 91.3 & \\
\hline Elevated $(>15 \mathrm{~mm} / \mathrm{h})$ & 24 & 47.9 & & 52.3 & \\
\hline Unknown & 19 & - & & - & \\
\hline
\end{tabular}

PFS: progression free survival; OS: overall survival; ALCL: Anaplastic large-cell lymphoma; ALK+: Anaplastic lymphoma kinase expressing; ALK-: without anaplastic lymphoma kinase expressing; PTCL-NOS: Peripheral T-cell lymphoma not otherwise specified; AITL: Angioimmunoblastic T-cell lymphoma; NKTCL: extranodal natural killer/T-cell lymphoma, nasal type; SPTCL: Subcutaneous panniculitis-like T-cell lymphoma; ECOG PS: Eastern Cooperative Oncology Group performance status; LDH: Lactate dehydrogenase; $\beta 2-M G$ : $\beta 2-$ Microglobulin; ESR: Erythrocyte sedimentation rate; aaIPI: age adjusted International Prognostic Index; PIT: Prognostic Index for T-cell lymphoma; HDT/ASCT: High-dose therapy and autologous stem cell transplantation; CR1: complete remission after first-line treatment; PR1: partial remission after first-line treatment; CR2: complete remission after second-line treatment; PR2: partial remission after second-line treatment; SD: Stable disease; PD: Progressive disease.

Table 4. Prognostic factors in multivariate analysis

\begin{tabular}{lllllll}
\hline & PFS & \multicolumn{5}{l}{ OS } \\
\hline & HR & $95 \%$ CI & $p$ & HR & $95 \%$ CI & $p$ \\
\hline $\begin{array}{l}\text { ESR before treatment } \\
\text { Response before }\end{array}$ & & & - & 0.184 & $0.022-1.503$ & 0.114 \\
HDT / ASCT & & & $\mathbf{0 . 0 0 1}$ & & & 0.247 \\
$\quad$ CR1 vs. SD/PD & 0.230 & $0.062-0.848$ & 0.027 & 0.123 & $0.011-1.344$ & 0.086 \\
$\quad$ PR1 vs. SD/PD & 0.218 & $0.026-1.836$ & 0.161 & 0.318 & $0.027-3.750$ & 0.362 \\
$\quad$ CR2 vs. SD/PD & 2.214 & $0.435-11.277$ & 0.339 & 0.000 & 0.000 & 0.988 \\
$\quad$ PR2 vs. SD/PD & 3.798 & $1.017-14.182$ & 0.047 & 1.644 & $0.260-10.412$ & 0.597 \\
LDH before HDT/ASCT & 0.275 & $0.077-0.980$ & $\mathbf{0 . 0 4 7}$ & & & - \\
R2-MG before & & & - & 1.177 & $0.175-7.934$ & 0.867 \\
HDT / ASCT & & & & & & \\
ESR before HDT/ASCT & 0.377 & $0.131-1.083$ & 0.070 & 0.756 & $0.147-3.900$ & 0.739
\end{tabular}

PFS: progression free survival; OS: overall survival; HR: Hazard risk; 95\% CI: 95\% confidence interval; NS: No statistical significance; ESR: Erythrocyte sedimentation rate; HDT / ASCT: High-dose therapy and autologous stem cell transplantation;

LDH: Lactate dehydrogenase; $\beta 2-M G$ : $\beta 2$-Microglobulin; CR1: complete remission after first-line treatment; PR1: partial remission after first-line treatment; CR2: Complete remission after second-line treatment; PR2: partial remission after second-line treatment; SD: Stable disease; PD: Progressive disease.

\section{EFS24, OSsub and survival in the entire cohort}

Among the 79 patients, 34 patients achieved EFS24, 11 patients died before the 24th month and 34 cases did not achieve EFS24. Therefore, OSsub data were only available from 68 patients with available EFS24 data. Median OSsub after progression within the first 24 months was 23.8 months, with an expected 3-year OSsub $47.8 \%$. In contrast, median OSsub after achieving EFS24 was 130.6 months, with an expected 3-year OSsub (total of 5 years after diagnosis) $100 \%$. Patients who achieved EFS24 had better outcomes than those failing to achieve EFS24 in OS (2-year OS: $91.1 \%$ vs. $50.6 \%, p<0.001)$.

\section{Safety and toxicity}

After HDT/ASCT, the median time to absolute neutrophil count recovery $\left(>0.5 \times 10^{9} / \mathrm{L}\right)$ was 10.8 days (6-14 days), and the median time to platelet recovery $\left(>20 \times 10^{9} / \mathrm{L}\right)$ was 12.1 days (7-25 days). Treatment- related complications included two cases of septicemia, one case of drug induced interstitial pneumonia, and one case of diarrhea due to gut microbiota imbalance, which were all successfully addressed. No patients died of treatment-related complications.

\section{Discussion}

Our study first showed that HDT/ASCT was a safe and effective regimen for both consolidation and salvage therapy in Chinese patients with PTCLs. The 2-year PFS and OS were $83.8 \%$ and $89.8 \%$ respectively for patients sensitive to first-line therapy in our study, while survival rates from past HDT/ASCT studies showed great variation among different trials.[13-16] As consolidation treatment, the largest prospective study $(\mathrm{N}=160)$ showed 5-year PFS $44 \%$ and 5-year OS 51\%.[16] The reported 3-year OS ranged from $44.5 \%$ to 73\% in other prospective studies.[13-15] The pooled OS rate in retrospective studies was $67.9 \%$ and the heterogeneity between studies was high $\left(\mathrm{I}^{2}=86.7 \%\right)$. [8] The survival rate in consolidation treatment group in our study was higher than previous reports. For relapse/refractory patients, the combination of chemotherapy and HDT/ASCT was the only available salvage therapy. Hence there were no prospective and controlled studies to confirm its efficacy. According to the meta-analysis about the efficacy of HDT/ASCT in relapse/refractory patients, the pooled PFS and OS rates were $36 \%$ and $47 \%$ respectively $\left(\mathrm{I}^{2}=0 \%\right)$.[8] In our study, the 2 -year PFS and OS were $32.9 \%$ and $50.5 \%$ respectively, which was similar with previous studies.

To explore the survival advantage of patients sensitive to first-line treatment, we compared their clinical features with those in previous studies. There was a high proportion of CR1 (38/47) in our patients. We showed a strong correlation between PFS/OS and the response before HDT/ASCT. Group CR1 (2-year PFS $85.8 \%$, 2-year OS 94.2\%) were superior to other groups in survival, which corroborated with other studies.[16-20] Given the absence of randomized controlled trial however, it is still uncertain about the clinical benefit of HDT/ASCT for CR1 patients, as they might get similar survival without HDT/ASCT. In group PR1, HDT/ASCT was clearly of great value in stopping or delaying disease progression. For patients in group CR2, who often did not respond well to first-line treatment and did not undergo HDT / ASCT before disease progression, their PFS was lower than that of PR1 patients who took HDT/ASCT immediately after first-line treatment. For relapse/ refractory patients, HDT/ASCT was only beneficial to patients who achieved CR in salvage therapy, but not to patients with PR2 or SD/PD. 
We then focused on the differential response to HDT/ASCT among pathological subtypes, especially in AITL and NKTCL. In patients with AITL, the survival rate (2-year PFS 74.3\%, 2-year OS 94.9\%) was superior to any other pathologic subtypes in our study, and was also greater than the average survival of AITL reported previously. AITL patients from the Group d'Etude des Lymphomes de l'Adulte (GELA) had a 2 -year OS $51 \%$, 5-year OS 33\%, and a plateau after 6 years.[21] The International Peripheral T-Cell Lymphoma Project also reported 5-year OS 32\% in AITL.[2] We attribute the considerable discrepancy between our data and previous results to the difference in patient conditions. AITL patients in previous studies were primarily elderly people. Infection was not uncommon, which often lead to premature termination of chemotherapy. For those who completed the first-line treatment, remission were often short-lived.[22] Previous reports showed that HDT/ASCT could increase the 5-year OS to $44 \%$, but it was associated a higher relapse rate $(50 \%$ at 2 years) as consolidation treatment after first-line therapy.[23] On the other hand, patients in our AITL group had age below 60. Chemotherapy was well tolerated and infection was rare. After first-line therapy, ten patients were sensitive to chemotherapy, $80 \%$ of whom achieved CR1. Since good chemosensitivity correlated with good survival after HDT/ASCT,[24, 25] it is not unexpected to have unusually high survival rates. Notwithstanding, some patients in our study might relapse in the future, as our median follow-up time was only 23.6 months.

In NKTCL, survival beyond 1 year was uncommon except for those with early-stage nasal disease, and the aggressive clinical behavior of primary extranasal disease is similar to that of advanced nasal disease.[12] Previous reports had showed the 5-year OS of all types NKTCLs, nasal disease, and extranasal disease were $32 \%, 42 \%, 9 \%$.[2, 12] All of 21 NKTCL patients in our study had at least one of risk factors as followed: relapse or refractory, advanced stage, and extranasal disease. The 2-year PFS and OS of them were $54.5 \%$ and $60.1 \%$, which were much better than previous reports. Most patients with CR1 (9/11) kept long time survival, while $70 \%$ non-CR1 patients suffered disease progression after HDT/ASCT. In patients who achieved CR1, previous retrospective analyses also showed advantage of HDT/ASCT on improving OS compared with historical matched controls, while such effect was absent in non-CR1 patients.[18, 26] All these evidences suggest that newly diagnosed advanced-stage nasal NKTCL and extranasal disease should receive HDT/ASCT as consolidation treatment, especially when they achieved CR after first-line treatment. Moreover, there were two clinical features in patients with progression disease after HDT/ASCT in our study: 1) disease progressed within 6 months and the survival curve showed an apparent plateau after 1 year, which were also shown by previous studies; $[27,28] 2) 88.9 \%$ (8/9) patients relapsed with newly diagnosed bone marrow involvement. To address this newly revealed issue of bone marrow involvement, we are now using TBI in combination with HD-CTX as conditioning regimen, instead of chemotherapy alone. Given the good sensitivity of NKTCL to radiotherapy, we anticipate this method to prolong PFS and improve OS.

Furthermore, our results corroborated with the report/study of Maurer MJ et al., [11] that EFS24 is a great prognostic indicator for survival. Patients who achieved EFS24 had better survival than those who did not (median OSsub: 130.6mon vs. 23.8mon; 2-year OS: $91.1 \%$ vs. $50.6 \%, p<0.001)$. As such, we recommend that EFS24 be adopted for future assessment of OS in PTCLs with HDT/ASCT.

In conclusion, HDT/ASCT was a safe and effective regimen for both consolidation and salvage therapy in Chinese patients with PTCLs. Response before HDT/ASCT was strongly correlated with PFS and OS. CR1 patients were superior to others in survival. PFS of CR2 patients was lower than that of PR1 patients who took HDT/ASCT immediately after first-line treatment. The efficacy of HDT/ ASCT varies among different pathological subtypes. AITL, newly diagnosed advanced-stage nasal NKTCL and extranasal disease who received HDT/ASCT after CR1 may have benefit in survival from HDT/ASCT. The future prospective trial is warranted to find appropriate patients who can get the benefit from HDT/ASCT.

\section{Acknowledgements}

This study was funded by the National Natural Science Foundation of China (No. 81470368 and 81670187), Beijing Natural Science Foundation (No. 7172047), Capital's Funds for Health Improvement and Research (No. 2018-1-2151), Beijing Municipal Administration of Hospitals' Ascent Plan (No. DFL20151001) and Beijing Municipal Administration of Hospitals Clinical Medicine Development of special funding support (No. XMLX201503).

\section{Competing Interests}

The authors have declared that no competing interest exists.

\section{References}

1. Listed N. A clinical evaluation of the International Lymphoma Study Group classification of non-Hodgkin's lymphoma. The Non-Hodgkin's Lymphoma Classification Project. Blood. 1997; 89: 3909-18. 
2. Vose J, Armitage J, Weisenburger D. International Peripheral T-Cell and Natural Killer/T-Cell Lymphoma Study: Pathology Findings and Clinical Outcomes. Journal of Clinical Oncology. 2008; 26: 4124-30.

3. Sun J, Yang Q, Lu Z, He M, Gao L, Zhu M, et al. Distribution of lymphoid neoplasms in China: analysis of 4,638 cases according to the World Health Organization classification. Am J Clin Pathol. 2012; 138: 429-34.

4. Adams SV, Newcomb PA, Shustov AR. Racial Patterns of Peripheral T-Cell Lymphoma Incidence and Survival in the United States. J Clin Oncol. 2016; 34: 963-71.

5. Zhang $\mathrm{Y}, \mathrm{Xu} \mathrm{W}$, Liu H, Li J. Therapeutic options in peripheral $\mathrm{T}$ cell lymphoma. J Hematol Oncol. 2016; 9: 37.

6. Foss F. Hematology: Relapsed and refractory PTCL-into the therapeutic abyss. Nature Reviews Clinical Oncology. 2011.

7. Tse E, Kwong Y-L. The diagnosis and management of NK/T-cell lymphomas. Journal of Hematology \& Oncology. 2017; 10.

8. Elasmar J, Reljic T, Ayala E, Hamadani M, Nishihori T, Kumar A, et al. Efficacy of high-dose therapy and autologous hematopoietic cell transplantation in peripheral T-cell lymphomas as front-line consolidation or in the relapsed/refractory setting: a systematic review/meta-analysis. Biology of Blood \& Marrow Transplantation Journal of the American Society for Blood \& Marrow Transplantation. 2015; 22: 802-14.

9. Swerdlow SH, Campo, E., Harris, N.L., Jaffe, E.S., Pileri, S.A., Stein, H., Thiele, J., Vardiman, J.W. WHO Classification of Tumours of Haematopoietic and Lymphoid Tissues. 2008.

10. Cheson BD, Pfistner B, Juweid ME, Gascoyne RD, Specht L, Horning SJ, et al. Revised response criteria for malignant lymphoma. Journal of Clinical Oncology Official Journal of the American Society of Clinical Oncology. 2007; 25: 579.

11. Maurer MJ, Ellin F, Srour L, Jerkeman M, Bennani NN, Connors JM, et al. International Assessment of Event-Free Survival at 24 Months and Subsequent Survival in Peripheral T-Cell Lymphoma. Journal of Clinical Oncology Official Journal of the American Society of Clinical Oncology. 2017: JCO2017738195.

12. Au WY, Weisenburger DD, Intragumtornchai $T$, Nakamura S, Kim WS, Sng I, et al. Clinical differences between nasal and extranasal natural killer/T-cell lymphoma: a study of 136 cases from the International Peripheral T-Cell Lymphoma Project. Blood. 2009; 113: 3931.

13. Rodriguez J, Conde E, Gutierrez A, Arranz R, Leon A, Marin J, et al. Frontline autologous stem cell transplantation in high-risk peripheral T-cell lymphoma: a prospective study from The Gel-Tamo Study Group. Eur J Haematol. 2007; 79: 32-8.

14. Nickelsen M, Ziepert M, Zeynalova S, Glass B, Metzner B, Leithaeuser M, et al. High-dose CHOP plus etoposide (MegaCHOEP) in T-cell lymphoma: a comparative analysis of patients treated within trials of the German High-Grade Non-Hodgkin Lymphoma Study Group (DSHNHL). Ann Oncol. 2009; 20: 1977-84.

15. Reimer P, Rudiger T, Geissinger E, Weissinger F, Nerl C, Schmitz N, et al. Autologous stem-cell transplantation as first-line therapy in peripheral T-cell lymphomas: results of a prospective multicenter study. J Clin Oncol. 2009; 27: 106-13.

16. d'Amore F, Relander T, Lauritzsen GF, Jantunen E, Hagberg H, Anderson H, et al. Up-front autologous stem-cell transplantation in peripheral $\mathrm{T}$-cell lymphoma: NLG-T-01. J Clin Oncol. 2012; 30: 3093-9.

17. Chen AI, McMillan A, Negrin RS, Horning SJ, Laport GG. Long-Term Results Of Autologous Hematopoietic Cell Transplantation For Peripheral T Cell Lymphoma: The Stanford Experience. Biology of Blood and Marrow Transplantation. 2008; 14: 741-7.

18. J L, WY A, MJ P, J S, S N, J K, et al. Autologous Hematopoietic Stem Cell Transplantation in Extranodal Natural Killer/T Cell Lymphoma: A Multinational, Multicenter, Matched Controlled Study. Biology of Blood \& Marrow Transplantation Journal of the American Society for Blood \& Marrow Transplantation. 2008; 14: 1356-64.

19. Gui L, Shi YK, He XH, Lei YH, Zhang HZ, Han XH, et al. High-dose therapy and autologous stem cell transplantation in peripheral T-cell lymphoma: treatment outcome and prognostic factor analysis. Int J Hematol. 2014; 99: 69-78.

20. Sims MC, Newell K, Eyre TA, Hatton C, Collins GP. Long-term outcomes of autologous stem cell transplantation for peripheral T-cell lymphomas across the Thames Valley (1997-2012). British Journal of Haematology. 2015; 169: 452-5.

21. Mourad N, Mounier N, Brière J, Raffoux E, Delmer A, Feller A, et al. Clinical, biologic, and pathologic features in 157 patients with angioimmunoblastic T-cell lymphoma treated within the Groupe d'Etude des Lymphomes de l'Adulte (GELA) trials. Blood. 2008; 111: 4463-70.

22. Dunleavy K, Wilson WH, Jaffe ES. Angioimmunoblastic T cell lymphoma: pathobiological insights and clinical implications. Current Opinion in Hematology. 2007; 14: 348 .

23. Mosalpuria K, Bociek RG, Vose JM. Angioimmunoblastic T-cell lymphoma management. Semin Hematol. 2014; 51: 52-8.

24. Rodriguez J, Conde E, Gutierrez A, Arranz R, Gandarillas M, Leon A, et al. Prolonged survival of patients with angioimmunoblastic T-cell lymphoma after high-dose chemotherapy and autologous stem cell transplantation: the GELTAMO experience. Eur J Haematol. 2007; 78: 290-6.

25. Kyriakou C, Canals C, Goldstone A, Caballero D, Metzner B, Kobbe G, et al. High-Dose Therapy and Autologous Stem-Cell Transplantation in Angioimmunoblastic Lymphoma: Complete Remission at Transplantation Is the Major Determinant of Outcome-Lymphoma Working Party of the European Group for Blood and Marrow Transplantation. Journal of Clinical Oncology. 2008; 26: 218-24.

26. Kim HJ, Bang SM, Lee J, Kwon HC, Suh C, Kim HJ, et al. High-dose chemotherapy with autologous stem cell transplantation in extranodal NK/T-cell lymphoma: a retrospective comparison with non-transplantation cases. Bone Marrow Transplantation. 2006; 37: 819-24.

27. Au WY, Lie AK, Liang R, Kwong YL, Yau CC, Cheung MM, et al. Autologous stem cell transplantation for nasal NK/T-cell lymphoma: a progress report on its value. Annals of Oncology. 2003; 14: 1673.

28. Fox CP, Boumendil A, Schmitz N, Finel H, Luan JJ, Sucak G, et al. High-dose therapy and autologous stem cell transplantation for extra-nodal NK/T lymphoma in patients from the Western hemisphere: a study from the European Society for Blood and Marrow Transplantation. Leuk Lymphoma. 2015; 56: 3295-300 